\title{
A Two-step Clustering Approach for Measuring Socioeconomic Factors Associated with Cardiovascular Health among Older Adults in South Korea
}

Lee, Chi-Young

Assistant Professor, School of Nursing \& Health Studies, University of Washington Bothell, Washington, USA

\begin{abstract}
Purpose: This study aimed to identify socioeconomic clusters of older adults and compare cardiovascular health among the identified clusters. Methods: A secondary analysis was performed using the data from 3,303 older adults (over 65 years of age; $56.5 \%$ women) who participated in the Korean National Health and Nutrition Examination Survey (2016 2017). A two-step cluster analysis was used to identify older adults' socioeconomic clusters based on 11 factors associated with Socioeconomic Status (SES). Differences in the cardiovascular health outcomes among the identified clusters were analyzed using the $x^{2}$ test and one-way ANOVA. Results: A three-cluster solution was selected $(p<.001)$ composed of low $(n=715)$, middle $(n=1,425)$, and high-SES clusters $(n=1,163)$. The three clusters differed significantly in the prevalence of diabetes $(p<.010)$, hypertension $(p<.001)$, and metabolic syndrome $(p<.001)$, with greater prevalence in the lower SES clusters. Similarly, systolic blood pressure $(p<.001)$, body mass index $(p<.010)$, and total cholesterol $(p<.010)$ differed significantly among the clusters in the same pattern. Conclusion: Older adults of lower SES clusters should be a crucial target group for health promotion interventions aimed at the prevention and management of cardiovascular disease risk factors. Tailored interventions can be developed by understanding intersecting SES risk factors in this group.
\end{abstract}

Key Words: Cluster analysis; Cardiovascular diseases; Socioeconomic factors; Health status disparities; Korea

\section{INTRODUCTION}

Socioeconomic Status (SES) is defined as the combined economic and social status of an individual, and health disparities disproportionately affecting people of lower SES have been a popular topic in the field of cardiovascular research [1]. Simultaneously, the validity of indicators measuring SES remains a major concern [1]. Although some published traditional indices such as income, education, and occupation have been responsible for successfully impacting a substantial proportion of cardiovascular events, it has been argued that no single parameter fully captures SES [2]. Notwithstanding, most studies in cardiovascular health employ a single SES predictor as a proxy for the effects of socioeconomic disadvantage $[3,4]$.

Using a single SES predictor oversimplifies the multifaceted and entwined dimensions of socioeconomic dis- advantage and may erroneously overestimate the impact of a variable, ultimately underestimating the true impact of social hierarchy on health outcomes [5]. Even when using other social variables as covariates, adjustments are likely to be incomplete and result in limited reliability [5]. In real-life scenarios, different SES indicators tend to cluster and intersect together [6], which underscores the theory of intersectionality. The theory of intersectionality suggests that various socially constructed categories of discrimination (i.e., SES, race or ethnicity, etc.) interact to contribute to health disparities [7]. Thus, there is a need for novel markers of SES developed through rigorous design and methods.

Cluster analysis can allow the meaningful interpretation of the complex concept of SES and facilitate the identification of a vulnerable group of low SES individuals [8]. Understanding the potential clustering of SES risk markers could facilitate an etiologic understanding of car-

Corresponding author: Lee, Chi-Young https://orcid.org/0000-0001-6860-452X

School of Nursing \& Health Studies, University of Washington Bothell, 18115 Campus Way NE, Bothell, WA 98011, USA.

Tel: +1-984-209-6277, Fax: +1-425-352-3237, E-mail: clee33@uw.edu

Received: Jul 17, 2020 / Revised: Nov 11, 2020 / Accepted: Nov 24, 2020

This is an open access article distributed under the terms of the Creative Commons Attribution Non-Commercial License (http://creativecommons.org/licenses/ by-nc/3.0), which permits unrestricted non-commercial use, distribution, and reproduction in any medium, provided the original work is properly cited. 
diovascular health and identify possible interventions that could address mutually reinforcing contributors to Cardiovascular Disease (CVD) [9]. Many western studies support the feasibility of applying clustering to assess various SES conditions and associated cardiovascular health [10,11]. For instance, Aungkulanon et al. [10] constructed a composite socioeconomic index using cluster analysis and explored the correlation between the composite score and cardiovascular mortality in Thailand. Additionally, Mirowsky et al. [11] examined residential-level SES clusters in central North Carolina with their clustering technique, which enables the examination of its relationship to various cardiovascular and metabolic biomarkers.

In South Korea (hereafter “Korea”), older adults are the most vulnerable population with greater socioeconomic disadvantages. A high percentage of the aging population is suffering from CVD or is at risk. Importantly, such cardiovascular health conditions are closely related to low SES $[12,13]$. However, no study has explored clusters of older adults based on various intersecting SES indicators or how clustering contributes to cardiovascular health in Korea. Although previous Korean studies employed a wide range of SES factors as predictors [14,15], each of which was investigated separately for its effect on cardiovascular health, this approach could obscure important social gradients in cardiovascular health.

\section{Aim of the Study}

Considering these challenges, this study aimed to classify older Korean adults into several socioeconomically homogeneous clusters based on various SES indicators and compare cardiovascular health among the identified clusters using cluster analysis. It is important to note that our analytic strategy was strongly informed by the theory of intersectionality. This theory helps researchers to be more explicit about why they include particular variables as important dimensions of disparity in studies [16].

In Korea, the literature on older adults includes different levels of SES indicators, including age, gender, financial and social resources, working status, welfare benefits, and living location, which are critical determinants that have implications for differential cardiovascular health risk $[12,13,17]$. Although the original research on intersectionality theory does not incorporate age, age was included due to its significant relation to the degree of physical and social privileges among older adults, which cannot be disaggregated when explicating the health in this group [18]. Occupation was also included because the employment rate in older Koreans is more than twice the
Organization for Economic Cooperation and Development (OECD) average [19]. Thus, we hypothesized that disparities in cardiovascular health among Korean older adults are embedded in relationships defined by intersections of the aforementioned indicators.

\section{METHODS}

\section{Design}

This was a secondary analysis of the cross-sectional data from the Korean National Health and Nutrition Examination Survey (KNHANES). This nationwide survey used a stratified, multistage, clustered probability sampling method to select a representative sample of the noninstitutionalized, civilian Korean population. KNHANES was selected because it provides comprehensive and detailed information on SES, health behaviors, healthcare utilization, anthropometric measures, and clinical profiles for CVD, all relevant to this study. KNHANES is composed of three distinct sections: a health interview survey, a health examination survey, and a nutrition survey for dietary assessment. This study uses the health interview and health examination survey.

\section{Ethical Considerations}

KNHANES is publicly available deidentified data which do not include personal information. However, this study received the approval of the Institutional Review Board of the institution where the corresponding author was affiliated (protocol number: Pro00103112).

\section{Analysis Sample}

Given the rapid transformation of social structures in Korea, we used the most recent survey $(2016,2017)$ to ensure the information is current. The data from each collection year was merged to create the study dataset. The study population was limited to adults aged 65 years or older. Among older adults who responded to the survey in 2016 and 2017, a total of 3,303 people participated in all three components (i.e. health interview, health examination, and nutrition survey); their data were analyzed in this study.

\section{Measurements}

1) Socioeconomic status.

Socioeconomic characteristics were measured using 
multiple questions on age (65 74 years or $\geq 75$ years), gender (men or women), income level (four quartiles; low, middle-low, middle-high, or high), having private insurance (yes or no), homeownership (yes or no), marital status (married/partnered or nonmarried/unpartnered), living arrangement ( $1 \mathrm{vs} \geq 2$ people in the household), working status (employed or unemployed), the recipients of the National Basic Livelihood Security System (NBLSS; yes or no), types of national insurance (self-employed insurance, employee insurance, or Korean Medicaid [a taxpayer-funded insurance program]), and geographic locations (urban, rural).

\section{2) Cardiovascular health.}

We operationalized the term cardiovascular health as a broad range of cardiovascular health outcomes, including CVD risk factors and the prevalence of metabolic syndrome and CVD.

CVD risk factors. These include Body Mass Index (BMI $\left[\mathrm{kg} / \mathrm{m}^{2}\right]$ ) equal to and over $25 \mathrm{~kg} / \mathrm{m}^{2}$ (obese; yes or no), waist circumference ( $\mathrm{cm}$; continuous), diabetes (yes or no), fasting glucose (mg/dL; continuous), HbA1c (\%; continuous), hypertension (yes or no), Systolic Blood Pressure (SBP [mmHg] continuous), dyslipidemia (yes or no), total cholesterol (mg/dL; continuous), High-Density Lipoprotein-Cholesterol (HDL-C [mg/dL]; continuous), triglyceride (mg/dL; continuous). The cutoff point for obesity (BMI $\geq 25 \mathrm{~kg} / \mathrm{m}^{2}$ ) was defined by the International Obesity Task Force for Asian Adults in the Asian and Pacific regions [20].

Prevalence of metabolic syndrome. The presence of metabolic syndrome was assessed based on the National Cholesterol Education Program criteria (NCEP, Adult Treatment Protocol, ATP-III) [21]. Participants with three of more of the following parameters were considered as having metabolic syndrome: waist circumference $\geq 90 \mathrm{~cm}$ in men and $\geq 80$ $\mathrm{cm}$ in women; fasting glucose $\geq 110 \mathrm{mg} / \mathrm{dL}$ or under treatment for diabetes; blood pressure $\geq 130 / 85 \mathrm{mmHg}$ or use of antihypertensive medication; HDL-C $<40 \mathrm{mg} / \mathrm{dL}$ in men and $<50 \mathrm{mg} / \mathrm{dL}$ in women; or triglyceride $\geq 150$ $\mathrm{mg} / \mathrm{dL}$.

Prevalence of CVD. This is a dichotomized variable, with the answer being either yes or no; CVD includes angina pectoris, stroke, or myocardial infarction.

\section{Statistical Analysis}

The cluster analysis procedure was conducted using SPSS, v.20 (SPSS Inc., Chicago, Illinois) to identify groups of older adults with distinct patterns of socioeconomic characteristics using 11 SES factors as categorical variables. A two-step cluster analysis was chosen because it is a scalable cluster analysis algorithm designed to handle large data sets with mixed continuous and categorical data. Following the procedures outlined by Norušis [22], the number of clusters was based on the best combination of low Bayesian Information Criterion (BIC), a high ratio of distance measures, and a high ratio of BIC changes. SES levels were attributed to clusters of people based on the author's interpretation of which indicators were more present in each cluster. To validate the cluster solution, $x^{2}$ (or Fisher's exact test, as appropriate) test was used (the cluster as the independent variable and socioeconomic characteristics as the dependent variables).

Differences in the cardiovascular health outcomes among the identified clusters were analyzed using the $x^{2}$ test for categorical variables and one-way ANOVA for continuous variables with a Tukey's post hoc test when necessary. All analyses were performed after considering primary sampling units, stratification, and sample weights which ensure the analysis results are representative of the target population. Missing value analysis was performed on each scale considered for analysis, as the two-step method does not tolerate missing values and would have excluded any sample with missing values [22]. Cases with missing data points were relatively few $(<15 \%$ for all variables); thus, the single imputation technique was used to preserve sample size. Missingness of continuous variables was replaced with mean substitutions. Missing values among categorical variables were replaced by the most common response. A two-tailed t-test with $\alpha=.050$ was used to assess statistical significance.

\section{RESULTS}

\section{Sociodemographic Characteristics of Participants by Cluster}

Table 1 summarizes the participants' sociodemographic characteristics by cluster. $\mathrm{A}$ three-cluster solution $\mathrm{BIC}=$ $26,530.16$, a ratio of distance measures $=1.62$, a ratio of BIC changes $=0.60)$ was selected $(p<.001)$ : high $(\mathrm{n}=715$; cluster $1)$, middle $(n=1,425$; cluster 2$)$, and low-SES clusters $(n=$ 1,163; cluster 3). Adults among the low-SES cluster were older than among the other two; this is explained by the largest proportion of adults aged over 75 years $(56.7 \%$ in this cluster vs. $40.0 \%$ in the middle-SES and $18.3 \%$ in the high-SES clusters. Participants in this cluster were more likely to be women $(82.7 \%)$ compared to the other two clusters. 
Table 1. Sociodemographic Characteristics of Participants by Cluster

$(N=3,303)$

\begin{tabular}{|c|c|c|c|c|c|c|c|}
\hline \multirow[t]{2}{*}{ Characteristics } & \multirow[t]{2}{*}{ Categories } & $\begin{array}{c}\text { Cluster } 1^{\dagger} \\
(\mathrm{n}=715)\end{array}$ & $\begin{array}{l}{\text { Cluster } 2^{\ddagger}}_{(n=1,425)} \\
\end{array}$ & $\begin{array}{l}{\text { Cluster } 3^{\S}}_{(n=1,163)} \\
\end{array}$ & $\begin{array}{c}\text { Total } \\
(n=3,303)\end{array}$ & \multirow[t]{2}{*}{$x^{2}$} & \multirow[t]{2}{*}{$p$} \\
\hline & & $\mathrm{n}(\%)$ & $\mathrm{n}(\%)$ & $\mathrm{n}(\%)$ & $\mathrm{n}(\%)$ & & \\
\hline \multirow[t]{2}{*}{ Age (year) } & $65 \sim 74$ & $584(81.7)$ & $855(60.0)$ & $503(43.3)$ & $1,942(58.8)$ & \multirow[t]{2}{*}{244.78} & \multirow[t]{2}{*}{$<.001$} \\
\hline & $\geq 75$ & 131 (18.3) & $570(40.0)$ & $660(56.7)$ & 1,361 (41.2) & & \\
\hline \multirow[t]{2}{*}{ Gender } & Men & $481(67.3)$ & 758 (53.2) & $200(17.2)$ & $1,439(43.6)$ & \multirow[t]{2}{*}{626.62} & \multirow[t]{2}{*}{$<.001$} \\
\hline & Women & $234(32.7)$ & $667(46.8)$ & $963(82.8)$ & $1,864(56.4)$ & & \\
\hline \multirow[t]{4}{*}{ Income } & High & $185(25.9)$ & 448 (31.4) & $191(16.4)$ & 824 (24.9) & \multirow[t]{4}{*}{97.28} & \multirow[t]{4}{*}{$<.001$} \\
\hline & Middle-high & $211(29.5)$ & $355(24.9)$ & $242(20.8)$ & $808(24.5)$ & & \\
\hline & Middle-low & $198(27.7)$ & $291(20.4)$ & $337(29.0)$ & $826(25.0)$ & & \\
\hline & Low & $121(16.9)$ & 331 (23.3) & $393(33.8)$ & 845 (25.6) & & \\
\hline \multirow{2}{*}{$\begin{array}{l}\text { Having private } \\
\text { insurance }\end{array}$} & Yes & $412(57.6)$ & $549(38.5)$ & $300(25.8)$ & $1,261(38.2)$ & \multirow[t]{2}{*}{192.51} & \multirow[t]{2}{*}{$<.001$} \\
\hline & No & $303(42.4)$ & $876(61.5)$ & $863(74.2)$ & $2,042(61.8)$ & & \\
\hline \multirow[t]{2}{*}{ House ownership } & Yes & $630(88.1)$ & $1,250(87.7)$ & $590(50.8)$ & $2,470(74.8)$ & \multirow[t]{2}{*}{365.04} & \multirow[t]{2}{*}{$<.001$} \\
\hline & No & 85 (11.9) & $175(12.3)$ & $573(49.2)$ & $833(25.2)$ & & \\
\hline \multirow[t]{2}{*}{ Marital status } & Married/partnered & 700 (97.9) & $1,328(93.2)$ & $176(15.1)$ & $2,204(66.7)$ & \multirow[t]{2}{*}{$2,249.43$} & \multirow[t]{2}{*}{$<.001$} \\
\hline & Nonmarried/unpartnered & $15(2.1)$ & $97(6.8)$ & $987(84.9)$ & $1,099(33.3)$ & & \\
\hline \multirow{2}{*}{$\begin{array}{l}\text { Living } \\
\text { arrangement }\end{array}$} & $\geq 2$ people in the household & $1,064(91.5)$ & 939 (65.9) & $489(68.4)$ & 2,492 (75.4) & \multirow[t]{2}{*}{281.02} & \multirow[t]{2}{*}{$<.001$} \\
\hline & Living alone & $99(8.5)$ & $486(34.1)$ & $226(31.6)$ & $811(24.6)$ & & \\
\hline \multirow[t]{2}{*}{ Working status } & Employment & $715(100.0)$ & $314(22.0)$ & $0(0.0)$ & $971(29.4)$ & \multirow[t]{2}{*}{$2,351.80^{\|}$} & \multirow[t]{2}{*}{$<.001$} \\
\hline & Unemployment & $0(0.0)$ & $1,111(78.0)$ & $1,163(100.0)$ & $2,332(70.6)$ & & \\
\hline \multirow{2}{*}{$\begin{array}{l}\text { Recipients of } \\
\text { NBLSS }\end{array}$} & No & $713(99.7)$ & $1,279(89.7)$ & $775(66.6)$ & 2,767 (83.8) & \multirow[t]{2}{*}{$843.85^{\|}$} & \multirow[t]{2}{*}{$<.001$} \\
\hline & Yes & $2(0.3)$ & $146(10.3)$ & $388(33.4)$ & $536(16.2)$ & & \\
\hline \multirow{3}{*}{$\begin{array}{l}\text { Type of national } \\
\text { insurance }\end{array}$} & Self-employed insurance & $251(35.1)$ & $471(33.0)$ & $324(27.8)$ & $1,046(31.7)$ & 208.98 & $<.001$ \\
\hline & Employee insurance & $463(64.8)$ & $952(66.8)$ & $620(53.3)$ & $2,035(61.6)$ & & \\
\hline & Korean Medicaid & $1(0.1)$ & $2(0.2)$ & $220(18.9)$ & $223(6.7)$ & & \\
\hline Geographical & Urban & $574(80.3)$ & $966(67.8)$ & $706(60.7)$ & $2,246(68.0)$ & 48.95 & $<.001$ \\
\hline location & Rural & $141(19.7)$ & 459 (32.2) & 457 (39.3) & $1,057(32.0)$ & & \\
\hline
\end{tabular}

NBLSS=National Basic Livelihood Security System; ${ }^{\dagger}$ Cluster $1=$ High socioeconomic status group; ${ }^{\dagger}$ Cluster $2=$ Middle socioeconomic status group; ${ }^{\S}$ Cluster $3=$ Low socioeconomic status group; $"$ Fisher's exact test.

The low-SES cluster also included the largest proportion of people in the two poorest income quintiles (62.8\%), while the high-SES cluster reported living in every income quintile except for the poorest. The low-SES cluster reported the lowest rates of people with private health insurance (25.8\%) and homeownership (50.8\%); in contrast, the high-SES cluster had the highest rate of people with private health insurance (57.6\%) and homeownership (88.1\%) among the three clusters. The low-SES cluster included the lowest proportion of married people $(15.1 \%)$ and the highest proportion of people living alone (31.6\%). No participants in the low-SES cluster were currently employed, whereas a high number of employed people were represented in the middle-SS $(22.0 \%)$ and high-SES clusters $(100.0 \%)$.

Older adults in the low-SES cluster were more likely to be recipients of NBLSS than those in the other two clusters (33.4\% in this cluster vs. $10.3 \%$ in the middle-SES and $0.3 \%$ in the high-SES clusters). They exhibited the highest proportion of Korean Medicaid beneficiaries (18.9\%) compared to the other two clusters $(0.2 \%$ and $0.1 \%$, for middle and high, respectively). They also reported the highest proportion of people living in rural settings (39.4\%) compared to those in middle-SES (32.2\%) and high-SES (19.7\%) clusters.

\section{Cardiovascular Health Outcomes of Participants by Cluster}

Significant differences in cardiovascular health outcomes were noted among the clusters (Table 2). The three clusters differed significantly in the prevalence of diabetes 
Table 2. Cardiovascular Health Outcomes of Participants by Cluster

$(N=3,303)$

\begin{tabular}{|c|c|c|c|c|c|c|c|}
\hline \multirow[t]{2}{*}{ Characteristics } & \multirow[t]{2}{*}{ Categories } & $\begin{array}{c}\text { Cluster } 1^{\dagger} \\
(\mathrm{n}=715)\end{array}$ & $\begin{array}{l}{\text { Cluster } 2^{\dagger}}_{(\mathrm{n}=1,425)}\end{array}$ & $\begin{array}{l}{\text { Cluster } 3^{\S}}_{(n=1,163)} \\
\end{array}$ & \multirow[t]{2}{*}{$x^{2}$ or $\mathrm{F}$} & \multirow[t]{2}{*}{$p$} & \multirow[t]{2}{*}{ Tukey } \\
\hline & & $\mathrm{n}(\%)$ or $\mathrm{M} \pm \mathrm{SD}$ & $\mathrm{n}(\%)$ or $\mathrm{M} \pm \mathrm{SD}$ & $\mathrm{n}(\%)$ or $\mathrm{M} \pm \mathrm{SD}$ & & & \\
\hline BMI & $\geq 25 \mathrm{~kg} / \mathrm{m}^{2}$ & $234(32.7)$ & $504(35.4)$ & $468(40.2)$ & 15.65 & $<.001$ & \\
\hline Waist circumference $(\mathrm{cm})$ & & $85.19 \pm 8.42$ & $86.85 \pm 9.42$ & $83.85 \pm 9.47$ & 0.48 & .742 & \\
\hline Diabetes prevalence & $\begin{array}{l}\text { Yes } \\
\text { No }\end{array}$ & $\begin{array}{l}141(19.7) \\
574(80.3)\end{array}$ & $\begin{array}{c}362(25.4) \\
1063(74.6)\end{array}$ & $\begin{array}{l}851(73.2) \\
312(26.8)\end{array}$ & 12.75 & .002 & \\
\hline Fasting glucose (mg/dL) & & $108.87 \pm 28.28$ & $108.42 \pm 26.96$ & $110.09 \pm 27.25$ & 1.22 & .295 & \\
\hline $\mathrm{HbA1c}(\%)$ & & $6.01 \pm 0.92$ & $6.04 \pm 0.90$ & $6.07 \pm 0.79$ & 1.17 & .310 & \\
\hline Hypertension prevalence & $\begin{array}{l}\text { Yes } \\
\text { No }\end{array}$ & $\begin{array}{l}327(45.7) \\
388(54.3)\end{array}$ & $\begin{array}{l}783(55.0) \\
642(45.0)\end{array}$ & $\begin{array}{l}706(60.7) \\
457(39.3)\end{array}$ & 40.09 & $<.001$ & \\
\hline SBP (mmHg) & & $127.82 \pm 16.77^{\mathrm{a}}$ & $127.29 \pm 16.37^{\mathrm{b}}$ & $130.30 \pm 18.07^{\mathrm{c}}$ & 9.28 & $<.001$ & $c>a, b$ \\
\hline $\begin{array}{l}\text { Dyslipidemia } \\
\text { prevalence }\end{array}$ & $\begin{array}{l}\text { Yes } \\
\text { No }\end{array}$ & $\begin{array}{l}167(23.4) \\
548(76.6)\end{array}$ & $\begin{array}{c}379(26.6) \\
1,046(73.4)\end{array}$ & $\begin{array}{l}309(26.6) \\
854(73.4)\end{array}$ & 3.04 & .218 & \\
\hline Total cholesterol (mg/dL) & & $183.56 \pm 36.90^{\mathrm{a}}$ & $184.49 \pm 36.87^{\mathrm{b}}$ & $188.87 \pm 38.63^{\mathrm{c}}$ & 6.83 & .001 & $c>a, b$ \\
\hline HDL-C (mg/dL) & & $48.40 \pm 11.65$ & $48.24 \pm 11.95$ & $47.51 \pm 10.76$ & 2.18 & .113 & \\
\hline Triglyceride (mg/dL) & & $135.52 \pm 88.92$ & $136.17 \pm 72.67$ & $138.75 \pm 71.62$ & 0.51 & .600 & \\
\hline Metabolic syndrome $\|$ & $\begin{array}{l}\text { Yes } \\
\text { No }\end{array}$ & $\begin{array}{l}215(30.1) \\
500(69.9)\end{array}$ & $\begin{array}{l}434(30.5) \\
991(69.5)\end{array}$ & $\begin{array}{l}488(42.0) \\
675(58.0)\end{array}$ & 45.23 & $<.001$ & \\
\hline CVD prevalence & $\begin{array}{l}\text { Yes } \\
\text { No }\end{array}$ & $\begin{array}{c}66(9.2) \\
649(90.8)\end{array}$ & $\begin{array}{c}158(11.1) \\
1267(88.9)\end{array}$ & $\begin{array}{c}117(10.1) \\
1046(89.9)\end{array}$ & 1.91 & .385 & \\
\hline
\end{tabular}

$\mathrm{BMI}=$ body mass index; $\mathrm{CVD}=$ cardiovascular disease; $\mathrm{HDL}-\mathrm{C}=$ high-density lipoprotein-cholesterol; $\mathrm{M}=$ mean; $\mathrm{SBP}=$ systolic blood pressure; $\mathrm{SD}=$ standard deviation; ${ }^{\dagger}$ Cluster $1=$ High socioeconomic status group; ${ }^{\dagger}$ Cluster $2=$ Middle socioeconomic status group; ${ }^{\S} \mathrm{Cluster} 3=$ Low socioeconomic status group; " The presence of metabolic syndrome was based on the NCEP ATP III guidelines.

$(p<.010)$, hypertension $(p<.001)$, and metabolic syndrome $(p<.001)$, with greater prevalence in the lower SES clusters. Similarly, SBP $(p<.001)$, BMI $(p<.010)$, and total cholesterol $(p<.010)$ differed significantly among the clusters in the same pattern. However, no significant differences were observed in the prevalence of dyslipidemia, waist circumference, fasting glucose, $\mathrm{HbA1c}$, HDL-C, triglyceride, and the prevalence of CVDs among clusters.

\section{DISCUSSION}

Using cluster analysis, older adults were categorized into low-, middle-, and high-SES clusters. Older adults of lower SES clusters exhibited significantly higher CVD risk factors. Importantly, our approach provided a detailed overview of important SES factors that mutually contribute to CVD risk. For instance, we demonstrated that lower SES clusters comprised older adults who lived at the intersection of less favorable material conditions (e.g. low in- come, no home ownership, no private insurance, or unemployed) had adverse outcomes, which highlights "the material hypothesis" [23,24]. This approach differs from early Korean studies of older adults which tended to report health outcomes of those in income poverty vs. those above the poverty line [12,13]; these studies overlooked the significance of material resources for explicating the health of this population group, which protected household budgets from expensive care and ensured access to healthcare.

Among the material factors that contributed to adverse cardiovascular health, and when combined with other SES risk factors, the findings on employment was particularly noticeable: no individuals in the low-SES cluster were employed at the time of the survey, whereas those in the high-SES cluster were all employed. Although Korea's average age of labor market exit is the highest among OECD countries at 72.90 years for men and 73.10 years for women [19], unemployment among older adults remains 
a burgeoning problem despite the recognized value of this demographic in Korean society [25]. Thus, our results indicate the need to urgently address the impact of unemployment on CVD risk, especially among older adults in the low-SES cluster identified in our analysis.

The study included marital status and living arrangements (i.e. indices of social networks and support) as SES factors and demonstrated that those who were nonmarried/unpartnered or lived alone mostly belonged to the low-SES cluster and had higher CVD risk factors. Studies emphasizing this "social-relational" aspect posits that health disparities cannot be entirely explained by well-known financial barriers or material factors [26,27]. Yet, such social-relational aspects as disparity factors relating to cardiovascular health among older individuals have not received much attention in research in Korean [13]. This discussion is important as an increasing number of older adults are living alone in Korea [28]. The actual remarriage or cohabitation rate among oldest-old adults remains very low due to traditional social norms and lower economic status [29]. Our findings demonstrate that the proportion of people aged 75 and over were more concentrated in the lower SES cluster.

This study included gender-related data for analysis and evaluated intersecting identities with other SES factors, which is a study design differing from most Korean studies of disparity that adopt separate gender analysis for research in this field [12,13]. Whilst there is no optimal research method for gender analysis, there is a growing awareness of the importance for research to move beyond simple binary thinking to consider how gender differences vary within specific situations or contexts; intersectionality is a way of thinking about this more complex profile of disparity [30]. Consequently, this study found that there are more women with multiple simultaneous socioeconomic disadvantages (e.g., no private insurance, unemployed, etc.) than men in the lower SES cluster-i.e., the group with significantly higher CVD risk factors. This agrees with the argument that connects an increased rate of cardiovascular events with the perception of "women's inferiority." Within patriarchal society, elderly Korean women have existed in a subservient position to men; this culture subordinates low-SES elderly Korean women socially and economically, prevents their pursuit of health resources, and makes them more vulnerable to facing undesirable health events [31]. Considering this situation in Korea with our findings emphasizes the need to improve the cardiovascular health of disadvantaged elderly women and ensure their health equity.

Clinically, intersecting SES risk factors identified in this study can suggest vulnerability markers, identifying individuals who need either more medical attention or closer follow-up care. This approach could also identify subgroups who would benefit from structural interventions, such as alleviating socioeconomic barriers to hospital access or specialized cardiovascular care. Further, the regression analyses revealed that the lower SES clusters have a significantly higher prevalence of diabetes, hypertension, total cholesterol, and obesity than the higher ones. Importantly, these CVD risk factors did not decrease in recent years, particularly in those with low SES in Korea [32]. This underscores the need to focus on improving screening, early detection, and treatment for patients with such risk factors. The prevalence of metabolic syndrome was significantly higher in this cluster. This additional finding on "integrated CVD risk" evaluation is also important, as existing clinical guidelines commonly recommend applying primary interventions according to the magnitude of the integrated risk [33].

In this study, we identified "upstream" SES factors that affect cardiovascular health simultaneously with individual-level factors: people on public assistance or Korean Medicaid or those who reside in rural areas constitute a high proportion of the lower SES clusters with higher CVD risk. Such results are important for Korea, where the government's socioeconomic support system for older adults remains unsatisfactory, which raises concerns regarding health-related issues [34]. The high outof-pocket payment in public assistance and limited benefit coverage in the Korean Medicaid system work as barriers to healthcare utilization, particularly in impoverished older adults. Additionally, the introduction of the Designated Doctor System to lessen Medicaid expenditures results in inconvenience in hospital use, particularly among the rural Medicaid beneficiaries [35]. From a policy standpoint, this provides strong support for policies that reduce overall CVD risk disparities.

We acknowledge several limitations of this study. First, our study design could not conclude causality between SES and cardiovascular health. Second, as some information was self-reported (e.g., SES, pre-existing medical conditions, etc.), we could not entirely exclude the possibility of response bias. Third, the results may not be widely applicable since SES conditions for older adults vary between different countries depending on their historical, social, and cultural contexts. Fourth, SES variables included in the secondary dataset potentially lacked depth because they were operationally defined by a single survey item or a subset of test items. For instance, unemployment does not necessarily reflect real income or welfare, 
for it can be argued that unemployed older adults today are materially better off than when fully employed. Additionally, living arrangements may include a range of relationship variations that are not reflected by any singular indicator. Fifth, education was not considered as the relative homogeneity of the sample concerning education variables (i.e., the majority of older adults belonged in the "little to no education" category), limiting our ability to detect education-related disparities in the outcome. Sixth, biomarkers reported in the dataset were measured at one point in time, which may affect the statistical validity of our conclusion. Last, the single imputation used in this study may reduce data variability, resulting in the underestimation of variances and standard errors.

Despite these limitations, this study demonstrates several strengths. First, an integrated socioeconomic indicator was developed and tested based on important SES indicators that adequately reflect disparities in cardiovascular health among older adults, which can be considered in future research. Second, the large sample size provided statistical power to determine robust relationships within clusters. Third, we used population-based data, significantly reducing the likelihood of selection bias. Last, this study provided preliminary evidence for the disparity mechanisms in CVD risk factors, which can suggest a clear role for the prevention of CVD morbidity and mortality, particularly for those with low SES.

\section{CONCLUSION}

Our study approach improved understanding of intersecting SES risk factors as associated with cardiovascular health among the older adult population. Importantly, we found out that CVD risk factors are significantly higher in the lower SES cluster which has a higher portion of those aged over 75 years, women, with a lower income, without private insurance and homeownership, single or living alone, unemployed, on public assistance, Korean Medicaid beneficiaries, and living in rural areas.

Our study has several implications for future research, clinical studies, and policy. First, studies targeting older adults should adopt fundamentally different conceptual approaches for measuring SES associated with cardiovascular health. Second, several intersecting SES risk factors identified in this study could be vulnerability indicators, identifying individuals who require more targeted screening, early detection, or focused medical attention. Last, the primary challenge for public professionals is to design tailored health policies that consider the socioeconomic variability within the older adult population.

\section{CONFLICTS OF INTEREST}

The author declared no conflict of interest.

\section{AUTHORSHIP}

Study conception and design acquisition, data collection, analyzing the data, and drafting and critical revision of the manuscript LC-Y.

\section{ACKNOWLEDGEMENT}

This study received the institutional review board approval from Duke University(protocol number: Pro00103112).

\section{REFERENCES}

1. Clark AM, DesMeules M, Luo W, Duncan AS, Wielgosz A. Socioeconomic status and cardiovascular disease: risks and implications for care. Nature Reviews Cardiology. 2009;6(11): 712-22. https://doi.org/10.1038/nrcardio.2009.163

2. Schultz WM, Kelli HM, Lisko JC, Varghese T, Shen J, Sandesara $\mathrm{P}$, et al. Socioeconomic status and cardiovascular outcomes: challenges and interventions. Circulation. 2018;137(20): 2166-78.

https://doi.org/10.1161/CIRCULATIONAHA.117.029652

3. Schumann B, Kluttig A, Tiller D, Werdan K, Haerting J, Greiser $\mathrm{KH}$. Association of childhood and adult socioeconomic indicators with cardiovascular risk factors and its modification by age: the CARLA Study 2002-2006. BMC Public Health. 2011; 11:289. https://doi.org/10.1186/1471-2458-11-289

4. Myers V, Gerber Y. Socioeconomic aspects of cardiovascular Health. In: Wakabayashi I, Groschner K, editors. Interdisciplinary concepts in cardiovascular health. Vienna: Springer; 2013. p. 187-203.

5. Adler N, Bush NR, Pantell MS. Rigor, vigor, and the study of health disparities. Proceedings of the National Academy of Sciences. 2012;109(Suppl 2):17154-9.

https://doi.org/10.1073/pnas.1121399109

6. Omorou AY, Coste J, Escalon H, Vuillemin A. Patterns of physical activity and sedentary behaviour in the general population in France: cluster analysis with personal and socioeconomic correlates. American Journal of Public Health. 2016;38(3):483-92. https://doi.org/10.1093/pubmed/fdv080

7. Winker G, Degele N. Intersectionality as multi-level analysis: dealing with social inequality. European Journal of Women's Studies. 2011;18(1):51-66.

https://doi.org/10.1177/1350506810386084

8. Cabieses B, Tunstall H, Pickett K. Understanding the socioeconomic status of international immigrants in Chile through hierarchical cluster analysis: a population-based study. International Migration. 2015;53(2):303-20.

https://doi.org/10.1111/imig.12077 
9. Everage NJ, Linkletter CD, Gjelsvik A, McGarvey ST, Loucks EB. Social and behavioral risk marker clustering associated with biological risk factors for coronary heart disease: NHANES 20012004. BioMed Research International. 2014;e389853.

https://doi.org/10.1155/2014/389853

10. Aungkulanon S, Tangcharoensathien V, Shibuya K, Bundhamcharoen K, Chongsuvivatwong V. Area-level socioeconomic deprivation and mortality differentials in Thailand: results from principal component analysis and cluster analysis. International Journal for Equity in Health. 2017;16:117. https://doi.org/10.1186/s12939-017-0613-z

11. Mirowsky JE, Devlin RB, Diaz-Sanchez D, Cascio W, Grabich $\mathrm{SC}$, Haynes C, et al. A novel approach for measuring residential socioeconomic factors associated with cardiovascular and metabolic health. Journal of Exposure Science \& Environmental Epidemiology. 2017;27(3):281-9. https://doi.org/10.1038/jes.2016.53

12. Lee CY, Im EO. Socioeconomic disparities in cardiovascular health in South Korea: a systematic review. Journal of Cardiovascular Nursing. 2021;36(1):8-22. https://doi.org/10.1097/JCN.0000000000000624

13. Lee CY, Lee YH. Measurement of socioeconomic position in research on cardiovascular heath disparity in South Korea: a systematic review. Journal of Preventive Medicine and Public Health. 2019;52(5):281-91.

https://doi.org/10.3961/jpmph.19.094

14. Khang YH, Kim HR. Socioeconomic Inequality in mortality using 12-year follow-up data from nationally representative surveys in South Korea. International Journal for Equity in Health. 2016;15:15-51. https://doi.org/10.1186/s12939-016-0341-9

15. Park SJ, Kang HT, Nam CM, Park BJ, Linton JA, Lee YJ. Sex differences in the relationship between socioeconomic status and metabolic syndrome: the Korean National Health and Nutrition Examination Survey. Diabetes Research and Clinical Practice. 2012;96(3):400-6.

https://doi.org/10.1016/j.diabres.2011.12.025

16. Warner LR. A best practices guide to intersectional approaches in psychological research. Sex Roles. 2008;59(5-6):454-63. https://doi.org/10.1007/s11199-008-9504-5

17. Lee C, Chee W, Im EO. TCTAP A-098 Inequalities in cardiovascular health among older adults in South Korea: the role of personal-, provider-and system-level factors. Journal of the American College of Cardiology. 2019;73(Suppl 15):S52.

18. Wang S. SES, social interaction, and health status. In: Hoshi T, Kodama S, editors. The structure of healthy life determinants. International Perspectives on Aging. Vol 18. Singapore: Springer; 2018. p. 83-102.

19. Organization for Economic Cooperation and Development (OECD). Working better with age: Korea. Paris: OECD Publishing; 2018 [cited 2020 June 10]. Available from: https://www.oecd-ilibrary.org/sites/9789264208261-en/index .html?itemId=/content/publication/9789264208261-en

20. World Health Organization. The Asia-Pacific perspective: redefining obesity and its treatment. IOTF, Health Communications Australia: Brisbane; 2000.

21. Cleeman JI, Grundy SM, Becker D, Clark L. Expert panel on detection, evaluation and treatment of high blood cholesterol in adults. Executive summary of the third report of the National Cholesterol Education Program (NCEP) Adult Treatment Panel (ATP III). Journal of the American Medical Association. 2001; 285(19):2486-97.

22. Norušis MJ. IBM SPSS statistics 19 statistical procedures companion. Vol 496. Upper Saddle River, NJ, USA: Prentice Hall; 2012.

23. Lynch JW, Smith GD, Kaplan GA, House JS. Income inequality and mortality: importance to health of individual income, psychosocial environment, or material conditions. British Medical Journal. 2000;320(7243):1200-4

24. Truesdale BC, Jencks C. The health effects of income inequality: averages and disparities. Annual Review of Public Health. 2016;37:413-30.

https://doi.org/10.1146/annurev-publhealth-032315-021606

25. Lee J, Kim MH. The effect of employment transitions on physical health among the elderly in South Korea: a longitudinal analysis of the Korean Retirement and Income Study. Social Science \& Medicine. 2017;181:122-30.

https://doi.org/10.1016/j.socscimed.2017.04.002

26. Uphoff EP, Pickett KE, Cabieses B, Small N, Wright J. A systematic review of the relationships between social capital and socioeconomic inequalities in health: a contribution to understanding the psychosocial pathway of health inequalities. International Journal for Equity in Health. 2013;12:54. https://doi.org/10.1186/1475-9276-12-54

27. Arcaya MC, Arcaya AL, Subramanian SV. Inequalities in health: definitions, concepts, and theories. Global Health Action. 2015; 8(1):27106. https://doi.org/10.3402/gha.v8.27106

28. Statistics Korea. Changes in the proportion of older adults living alone [Internet]. Seoul: Statistics Korea; 2020 [cited 2020 June 9]. Available from: https://www.index.go.kr/unify/idx-info.do?idxCd=4233

29. Lee MA. Effects of economic and health conditions on the transition to living alone: a longitudinal study on older Koreans. Development and Society. 2016;45(3):591-617.

30. Scott N, Siltanen J. Gender and intersectionality-A quantitative toolkit for analyzing complex inequalities. Employment and Social Development Canada; 2020 [cited 2020 June 10]. Available from:

http://www.janetsiltanen.ca/toolkitenglish.pdf

31. Laura CN, Cho H. Women, gender, and social change in South Korea since 1945. In: Seth MJ, editor. Routledge handbook of 
modern Korean history. Routledge: Taylor \& Francis Group; 2016. p. 326-39.

32. Hwang J, Lee EY, Lee CG. Measuring socioeconomic inequalities in obesity among Korean adults, 1998-2015. International Journal of Environmental Research and Public Health. 2019;16 (9):1617. https://doi.org/10.3390/ijerph16091617

33. Zhao D, Liu J, Xie W, Qi Y. Cardiovascular risk assessment: a global perspective. Nature Reviews Cardiology. 2015;12(5): 301-11. https://doi.org/10.1038/nrcardio.2015.28

34. Jeon B, Noguchi H, Kwon S, Ito T, Tamiya N. Disability, pov- erty, and role of the basic livelihood security system on health services utilization among the elderly in South Korea. Social Science \& Medicine. 2017;178:175-83.

https://doi.org/10.1016/j.socscimed.2017.02.013

35. Kim MJ, Cho YH, Park NH. The effects of the designated doctor system on health care utilization of medical aid beneficiaries with chronic diseases. Journal of Korean Academy of Community Health Nursing. 2015;26(3):278-91.

https://doi.org/10.12799/jkachn.2015.26.3.278 